\title{
Classical molecular dynamics simulations of crystal lattices with truncated Taylor series-based interatomic potentials
}

\author{
Shrikant KSHIRSAGAR, Kranthi K. MANDADAPU† and Panayiotis PAPADOPOULOS ${ }^{\ddagger}$
}

\section{Table of contents}

1 Introduction 2

2 Theory 4

2.1 Background on interatomic potentials . . . . . . . . . . . . . . 4

2.2 Range of validity . . . . . . . . . . . . . . . . . . . . . . . . 4

2.3 Stress and heat flux . . . . . . . . . . . . . . . . 6

3 Application to the LJ potential $\quad 6$

4 Simulation procedure 11

4.1 Implementation as an interatomic potential . . . . . . . . . . . . . . . . 11

4.2 Computation of specific heat capacity for solid Ar . . . . . . . . . . . 12

4.3 Computation of thermal conductivity for solid Ar . . . . . . . . . . . . . . 14

$\begin{array}{llr}5 & \text { Results } & 14\end{array}$

6 Conclusion $\quad 16$

Appendix A: An efficient algorithm for computing equilibrium positions in a crystal lattice

\begin{abstract}
This article discusses a general method for constructing interatomic potentials based on truncated Taylor series expansion. Specifically, it addresses the scope of application of the method, and demonstrates its practical importance in capturing anharmonicity for a Lennard-Jones solid. In particular, the third-order terms in the truncated potential are shown to accurately approximate the thermal conductivity of the standard interaction Lennard-Jones potential. The paper also describes an efficient algorithm for locating the equilibrium lattice site of an atom in a three-dimensional crystal lattice displaced from its equilibrium position.
\end{abstract}

Keywords: molecular dynamics; Taylor series; anharmonicity; Lennard-Jones; specific heat; Thermal conductivity

\footnotetext{
${ }^{*}$ Department of Mechanical Engineering, University of California, Berkeley

${ }^{\dagger}$ Department of Chemical and Biomolecular Engineering, University of California, Berkeley and Chemical Sciences Division, Lawrence Berkeley National Laboratory

${ }^{\ddagger}$ Department of Mechanical Engineering, University of California, Berkeley
} 


\section{Introduction}

Molecular Dynamics (MD) is an invaluable computational tool for the bridging of realand phonon-space analysis techniques. A key advantage of MD over conventional lattice dynamics methods is that it allows for the natural inclusion of anharmonic effects, which are crucial in estimating most thermophysical properties [1].

The application of MD to the quantitative analysis of material response and properties is predicated upon the availability of accurate and efficient potentials. In principle, such potentials are intended to represent interatomic interactions between charged atomic particles. While ab initio MD is possible, its scope is extremely limited due to its prohibitive computational cost. Moreover, potential energy surfaces, which are solutions of the electronic Schrödinger equation within the Born-Oppenheimer approximation, are not readily available for most interesting systems. For this reason, one typically relies on a coarse empirical approximation to the true quantum-mechanical potential. Ideally, empirical potentials should have a simple functional form to speed up the evaluation of the forces acting on an atom and also be transferable to many systems under different loading conditions. Thus, designing a general empirical interatomic potential that approximates the actual (unknown) solution of the Schrödinger equation is a challenging task. In practice, the choice of functional form and parameters is often based on fitting to available experimental data (e.g., equilibrium geometry of stable phases, cohesive energy, elastic moduli, vibrational frequencies, temperatures of the phase transitions, etc.). Unsurprisingly, a potential that is tailored to one set of experimental data for a given material does not necessarily predict with accuracy the response of the same material beyond the narrow scope of this data. For example, if a potential has been designed to reproduce mechanical properties, such as the experimental values of the lattice constant and elastic constants for a crystal phase, one should not expect it to accurately predict thermal transport properties [2].

Recent analytical work has introduced a general method of constructing interatomic potentials based on a truncated Taylor series expansion of the (unknown) potential function of the crystal to a given order [3,4]. The coefficients in the Taylor series may be obtained from a given interatomic potential or through $a b$ initio methods, e.g., Density Functional Theory (DFT) calculations. The latter enables the use of interatomic potentials of ab initio accuracy for MD simulations, thereby removing one of the major limitations of classical $\mathrm{MD}$, that is, the dependence on purely empirical potentials.

While past work $[3,4]$ focused on the application of the method of truncated Taylor se- 
ries potential, it did not include any information on the conditions under which this method is applicable. Further, it is not clear how many terms in the Taylor series expansion are required to accurately estimate thermophysical properties of crystal lattices, such as heat capacity and thermal conductivity, the latter of which depends strongly on the degree of anharmonicity of the potential energy. This is particularly important because inclusion of higher-order terms becomes computationally expensive in terms of storage of force constants. In addition, calculating these higher-order force constants for real materials using an $a b$ initio method such as DFT is extremely challenging. As a result, most DFT-based methods are currently limited to computation of terms up to the third-order in the Taylor series expansion.

This article expands on the previous work by addressing the range of applicability of the truncated Taylor series expansion method. It also demonstrates the effectiveness of this method in yielding accurate estimates of equilibrium properties such as heat capacity as well as transport properties such as thermal conductivity. The latter affirms the sufficiency of the third-order terms in representing the anharmonicity of crystal lattices, thereby clarifying the importance of the third-order terms in relation to higher-order terms in the potential energy expression of solid crystals. For simplicity, the method is illustrated for an ideal Lennard-Jones (LJ) crystal, as the closed functional form of an LJ solid allows for an accurate determination of the error incurred in the estimation of thermophysical properties by approximating the potential energy with a truncated Taylor series. It must be noted that the predictive capacity of this method is not limited to materials whose potential is explicitly known. Indeed, the real utility of this method is in enabling the use of interatomic potentials of $a b$ initio accuracy to make more accurate predictions of thermophysical properties for a range of temperatures.

The article is organized as follows: Section 2 includes an introduction of the fundamental concepts of the truncated Taylor series technique and addresses the conditions under which it may be applied. It also includes a section on the appropriate statistical mechanical expressions for stress and heat flux to be used with the truncated Taylor series potential. Details on the implementation of the method for the case of a Lennard-Jones (LJ) crystal are presented in Section 3. This is followed in Section 4 by a discussion of implementation and a comparison of specific heat and thermal conductivity values using this method and a classical LJ potential. Concluding remarks are offered in Section 6. 


\section{Theory}

\subsection{Background on interatomic potentials}

In any crystal at finite temperature, the atoms undergo small-amplitude vibrations about their equilibrium lattice positions. This allows the potential energy $U$ to be expanded in a Taylor series about its equilibrium value $U_{0}$ in terms of the displacements of the atoms from their equilibrium positions, in the form

$$
U=U_{0}+\sum_{i ; \alpha} \Pi_{i}^{\alpha} u_{i}^{\alpha}+\frac{1}{2 !} \sum_{i, j ; \alpha, \beta} \Phi_{i j}^{\alpha \beta} u_{i}^{\alpha} u_{j}^{\beta}+\frac{1}{3 !} \sum_{i, j, k ; \alpha, \beta, \gamma} \Psi_{i j k}^{\alpha \beta \gamma} u_{i}^{\alpha} u_{j}^{\beta} u_{k}^{\gamma}+\ldots
$$

Here, $u$ denotes the atomic displacement, $i, j, k$ label the different atoms and $\alpha, \beta, \gamma$ denote the Cartesian directions. In addition, the force constants (FCs) $\Pi_{i}^{\alpha}, \Phi_{i j}^{\alpha \beta}, \Psi_{i j k}^{\alpha \beta \gamma}$ denote respectively the first, second and third partial derivatives of $U$ with respect to the displacements evaluated in the equilibrium configuration. Without loss of generality, the constant term $U_{0}$ may be set to 0 by adjusting the reference level of the potential energy. Furthermore, $\Pi_{i}^{\alpha}=0$ since the equilibrium configuration corresponds to a minimum of the potential energy, Therefore, Equation (1) may be reduced to

$$
U=\frac{1}{2 !} \sum_{i, j ; \alpha, \beta} \Phi_{i j}^{\alpha \beta} u_{i}^{\alpha} u_{j}^{\beta}+\frac{1}{3 !} \sum_{i, j, k ; \alpha, \beta, \gamma} \Psi_{i j k}^{\alpha \beta \gamma} u_{i}^{\alpha} u_{j}^{\beta} u_{k}^{\gamma}+\ldots .
$$

The components $F_{i}^{\alpha}$ of the total force acting on atom $i$ may be obtained from Equation (2) as

$$
F_{i}^{\alpha}=-\frac{\partial U}{\partial u_{i}^{\alpha}}=-\sum_{j ; \beta} \Phi_{i j}^{\alpha \beta} u_{j}^{\beta}-\frac{1}{2 !} \sum_{j, k ; \beta, \gamma} \Psi_{i j k}^{\alpha \beta \gamma} u_{j}^{\beta} u_{k}^{\gamma}-\ldots
$$

For simple systems (e.g., an LJ crystal) the FCs may be calculated analytically. However, for many realistic systems, where highly accurate interatomic potentials are not known, ab initio methods based on DFT may be employed to compute the FCs in Equation (3). In this manner, one may, in principle, construct interatomic potentials of ab initio accuracy up to any given order.

\subsection{Range of validity}

Classical MD simulations are meaningful when the temperature is high enough to render the classical Maxwell-Boltzmann distribution of atomic velocities a good approximation to the corresponding quantum statistical distribution. This is the case when the temperature is at least in the range of the Debye temperature of the material [5, Chapter 23]. 
For the purpose of accurately estimating many thermophysical properties of materials, including transport properties such as thermal conductivity, it is sufficient to include the effect of anharmonicity of the potential and truncate the Taylor series in Equation (2) at the third order [4], as discussed in the Introduction. This truncation is predicated on the assumption that the displacement $\mathbf{u}$ of the atoms about their equilibrium positions is "small" in the sense that

$$
\max _{i, j}\left(\left\|\mathbf{u}_{i}\right\|,\left\|\mathbf{u}_{j}\right\|\right) \ll\left\|\mathbf{R}_{j}-\mathbf{R}_{i}\right\| \equiv\left\|\mathbf{R}_{i j}\right\|,
$$

for all pairs of atoms $(i, j)$ with equilibrium positions $\mathbf{R}_{i}$ and $\mathbf{R}_{j}$. This truncation also serves the purpose of limiting the computational expense of the resulting MD simulations. For solids, condition (4) is typically satisfied unless the temperature is close to the melting point.

The precise meaning of the inequality condition (4) may be articulated as follows: the temperature of the solid must remain low enough so that no atom displaces so far away from its equilibrium position that the resultant force on it is directed away from that position. Figure 1 illustrates, for the simple case of a single-variable potential, the existence of a critical displacement beyond which an initially restoring force on an atom becomes disturbing in the case of an anharmonic potential. It can be seen from the figure that while this issue does not arise for purely harmonic potentials, all higher-order approximations suffer from this phenomenon. In general, the addition of a fourth-order term has minimal effect on the critical displacement because: (a) the FCs are typically much smaller in magnitude than their third-order counterparts and (b) the correction provided by the fourth-order is only appreciable at displacements much larger than the critical one, where anyway inequality (4) is violated. Therefore, if there exists a critical displacement in the third-order truncation, then it will persist even in the presence of higher-order terms. Figure 1 further suggests this point by showing the effect of a fourth-order term to the the Taylor series expansion of a general potential energy for a crystal lattice.

In summary, the proposed method is most applicable in a range of temperatures bounded from below by the Debye temperature of the material and from above by the temperature for which condition (4) no longer holds for any given atom in the crystal. The latter temperature depends on the width of the interatomic potential function well at the global minimum corresponding to the equilibrium configuration and, hence, must be determined by trial-and-error for each material. 


\subsection{Stress and heat flux}

Following Irving and Kirkwood [6], the expressions for the stress tensor and the heat flux may be derived in terms of atomistic variables. This procedure was originally developed for pair potentials only. A suitable generalization which is compatible with the multibody potential in Equation (2) is derived in [7] and leads to the definition of the instantaneous macroscopic stress tensor $\tilde{\mathbf{T}}$ as

$$
\begin{aligned}
& \tilde{\mathbf{T}}=-\frac{1}{V} \sum_{i} m_{i}\left(\frac{\mathbf{p}_{i}}{m_{i}}-\mathbf{v}\right) \otimes\left(\frac{\mathbf{p}_{i}}{m_{i}}-\mathbf{v}\right)- \\
& \frac{1}{V}\left[\frac{1}{2 !} \sum_{i, j} \mathbf{F}_{i j} \otimes \mathbf{r}_{i j}+\frac{1}{3 !} \sum_{i, j, k} \mathbf{F}_{i j k} \otimes\left(\mathbf{r}_{i j}+\mathbf{r}_{i k}\right)+\ldots\right],
\end{aligned}
$$

where $\mathbf{p}_{i}$ and $m_{i}$ denote the momentum and mass of the $i$-th atom respectively, $\mathbf{v}=\frac{\sum_{i} \mathbf{p}_{i}}{\sum_{i} m_{i}}$ denotes the macroscopic velocity vector, and $\mathbf{r}_{i j}$ denotes the vector connecting the positions of the $i$-th and $j$-th atoms. Also, the forces $\mathbf{F}_{i j}$ and $\mathbf{F}_{i j k}$ are defined by

$$
\mathbf{F}_{i j}=-\frac{\partial}{\partial \mathbf{r}_{i}} u_{2}\left(\mathbf{r}_{i}, \mathbf{r}_{j}\right)
$$

and

$$
\mathbf{F}_{i j k}=-\frac{\partial}{\partial \mathbf{r}_{i}} u_{3}\left(\mathbf{r}_{i}, \mathbf{r}_{j}, \mathbf{r}_{k}\right)
$$

where $u_{2}\left(\mathbf{r}_{i}, \mathbf{r}_{j}\right)$ and $u_{3}\left(\mathbf{r}_{i}, \mathbf{r}_{j}, \mathbf{r}_{k}\right)$ denote the two- and three-body contributions to the

total potential energy, respectively. Similarly, the instantaneous macroscopic heat flux $\tilde{\mathbf{J}}_{Q}$ is given in $[8]$ as

$$
\tilde{\mathbf{J}}_{Q}=\frac{1}{V} \sum_{i}\left[e_{i} \mathbf{I}+\frac{1}{2 !} \sum_{j} \mathbf{r}_{i j} \otimes \mathbf{F}_{i j}+\frac{1}{3 !} \sum_{j, k}\left(\mathbf{r}_{i j}+\mathbf{r}_{i k}\right) \otimes \mathbf{F}_{i j k}+\ldots\right]\left(\frac{\mathbf{p}_{i}}{m_{i}}-\mathbf{v}\right),
$$

where $\mathbf{I}$ denotes the second-order identity tensor and $e_{i}$ denotes the total energy per atom, defined as

$$
e_{i}=\frac{\mathbf{p}_{i} \cdot \mathbf{p}_{i}}{2 m_{i}}+\frac{1}{2 !} \sum_{j} u_{2}\left(\mathbf{r}_{i}, \mathbf{r}_{j}\right)+\frac{1}{3 !} \sum_{j, k} u_{3}\left(\mathbf{r}_{i}, \mathbf{r}_{j}, \mathbf{r}_{k}\right)+\ldots
$$

\section{Application to the LJ potential}

The proposed technique of expanding potentials in a Taylor series is illustrated by application to a solid described by the Lennard-Jones interatomic potential. The simple analytical form of the LJ potential allows the exact computation of the expressions for the FCs in 
the Taylor series expansion up to a given order, thereby serving as a very good test model for this technique.

By way of background, recall that in an LJ solid the potential energy between two atoms $i$ and $j$, separated instantaneously by distance $r_{i j}$, is defined as

$$
U_{i j}\left(r_{i j}\right)=\frac{A}{r_{i j}^{12}}-\frac{B}{r_{i j}^{6}}
$$

where $A$ and $B$ are constants. These constants are related to the standard LJ parameters as $A=4 \epsilon \sigma^{12}$ and $B=4 \epsilon \sigma^{6}$, where $\epsilon$ and $\sigma$ are the standard LJ parameters. Consequently, the total potential energy of an LJ crystal is given by the sum of all pairwise interaction energies between the atoms in the crystal according to

$$
U=\frac{1}{2} \sum_{i} \sum_{j \neq i} U_{i j}
$$

where the factor $\frac{1}{2}$ compensates for the double-counting of the pairwise interactions.

To expand $U$ in a Taylor series as in Equation (1), it is first necessary to express $U_{i j}$ in terms of the displacements $\mathbf{u}_{i}$ and $\mathbf{u}_{j}$. This can be achieved by noting that

$$
r_{i j}^{2}=\left\|\mathbf{R}_{i j}+\mathbf{u}_{i j}\right\|^{2}=R_{i j}^{2}\left(1+2 \frac{\mathbf{R}_{i j}}{R_{i j}} \cdot \frac{\mathbf{u}_{i j}}{R_{i j}}+\frac{u_{i j}^{2}}{R_{i j}^{2}}\right)=R_{i j}^{2}\left(1+2 \frac{u_{i j}}{R_{i j}} \cos \theta_{i j}+\frac{u_{i j}^{2}}{R_{i j}^{2}}\right)
$$

where $\mathbf{u}_{i j}=\mathbf{u}_{j}-\mathbf{u}_{i}$ and $\theta_{i j}$ is the angle between $\mathbf{R}_{i j}$ and $\mathbf{u}_{i j}$, as shown in Figure 2. Substituting the expression for $r_{i j}$ in Equation (10) allows for expanding $U_{i j}$ in a Taylor 
series around $u_{i j}=0$. This series may be truncated at third order, leading to

$$
\begin{aligned}
U_{i j} & =\frac{A}{R_{i j}^{12}\left(1+2 \frac{u_{i j}}{R_{i j}} \cos \theta_{i j}+\frac{u_{i j}^{2}}{R_{i j}^{2}}\right)^{6}}-\frac{B}{R_{i j}^{6}\left(1+2 \frac{u_{i j}}{R_{i j}} \cos \theta_{i j}+\frac{u_{i j}^{2}}{R_{i j}^{2}}\right)^{3}} \\
& \doteq \frac{A}{R_{i j}^{12}}\left[1-6\left(2 \frac{u_{i j}}{R_{i j}} \cos \theta_{i j}+\frac{u_{i j}^{2}}{R_{i j}^{2}}\right)+\frac{(-6)(-7)}{2}\left(2 \frac{u_{i j}}{R_{i j}} \cos \theta_{i j}+\frac{u_{i j}^{2}}{R_{i j}^{2}}\right)^{2}\right. \\
+ & \left.\frac{(-6)(-7)(-8)}{6}\left(2 \frac{u_{i j}}{R_{i j}} \cos \theta_{i j}+\frac{u_{i j}^{2}}{R_{i j}^{2}}\right)^{3}\right]-\frac{B}{R_{i j}^{6}}\left[1-3\left(2 \frac{u_{i j}}{R_{i j}} \cos \theta_{i j}+\frac{u_{i j}^{2}}{R_{i j}^{2}}\right)\right. \\
+ & \left.\frac{(-3)(-4)}{2}\left(2 \frac{u_{i j}}{R_{i j}} \cos \theta_{i j}+\frac{u_{i j}^{2}}{R_{i j}^{2}}\right)^{2}+\frac{(-3)(-4)(-5)}{6}\left(2 \frac{u_{i j}}{R_{i j}} \cos \theta_{i j}+\frac{u_{i j}^{2}}{R_{i j}^{2}}\right)^{3}\right] \\
& =\left[\frac{A}{R_{i j}^{12}}-\frac{B}{R_{i j}^{6}}\right]+\left[-12 \frac{A}{R_{i j}^{12}} \cos \theta_{i j}+6 \frac{B}{R_{i j}^{6}} \cos \theta_{i j}\right] \frac{u_{i j}}{R_{i j}} \\
+ & {\left[\frac{A}{R_{i j}^{12}}\left(-6+84 \cos ^{2} \theta_{i j}\right)-\frac{B}{R_{i j}^{6}}\left(-3+24 \cos ^{2} \theta_{i j}\right)\right] \frac{u_{i j}^{2}}{R_{i j}^{2}} } \\
+ & {\left[\frac{A}{R_{i j}^{12}}\left(84 \cos \theta_{i j}-448 \cos ^{3} \theta_{i j}\right)-\frac{B}{R_{i j}^{6}}\left(24 \cos _{i j}-80 \cos ^{3} \theta_{i j}\right)\right] \frac{u_{i j}^{3}}{R_{i j}^{3}} . }
\end{aligned}
$$

Thus, the pairwise potential term $U_{i j}$ has been approximated in terms of ascending powers of $\frac{u_{i j}}{R_{i j}}$ in the form

$$
U_{i j}=U_{0, i j}+U_{1, i j}+U_{2, i j}+U_{3, i j} .
$$

According to Equation (2), the constant term $U_{0, i j}$ and the linear term $U_{1, i j}$ do not contribute to the total crystal potential energy, hence they are ignored. The quadratic term $U_{2, i j}$ may be compactly expressed as

$$
\begin{aligned}
U_{2, i j} & =\left[\frac{A}{R_{i j}^{12}}\left(-6+84 \cos ^{2} \theta_{i j}\right)-\frac{B}{R_{i j}^{6}}\left(-3+24 \cos ^{2} \theta_{i j}\right)\right] \frac{u_{i j}^{2}}{R_{i j}^{2}} \\
& =\left[\left(-\frac{6 A}{R_{i j}^{12}}+\frac{3 B}{R_{i j}^{6}}\right)+\left(\frac{84 A}{R_{i j}^{12}}-\frac{24 B}{R_{i j}^{6}}\right) \cos ^{2} \theta_{i j}\right] \frac{u_{i j}^{2}}{R_{i j}^{2}} \\
& =C_{i j} u_{i j}^{2}+D_{i j}\left(\mathbf{u}_{i j} \cdot \mathbf{R}_{i j}\right)^{2},
\end{aligned}
$$

where

$$
C_{i j}=-\frac{6 A}{R_{i j}^{14}}+\frac{3 B}{R_{i j}^{8}}
$$

and

$$
D_{i j}=\frac{84 A}{R_{i j}^{16}}-\frac{24 B}{R_{i j}^{10}}
$$


Likewise, the cubic terms $U_{3, i j}$ may be written as

$$
\begin{aligned}
U_{3, i j} & =\left[\frac{A}{R_{i j}^{12}}\left(84 \cos \theta_{i j}-448 \cos ^{3} \theta_{i j}\right)-\frac{B}{R_{i j}^{6}}\left(24 \cos \theta_{i j}-80 \cos ^{3} \theta_{i j}\right)\right] \frac{u_{i j}^{3}}{R_{i j}^{3}} \\
& =\left[\frac{84 A}{R_{i j}^{15}}-\frac{24 B}{R_{i j}^{9}}\right] u_{i j}^{2}\left(u_{i j} \cos \theta_{i j}\right)+\left[\frac{-448 A}{R_{i j}^{15}}+\frac{80 B}{R_{i j}^{9}}\right]\left(u_{i j} \cos \theta_{i j}\right)^{3} \\
& =D_{i j} u_{i j}^{2}\left(\mathbf{u}_{i j} \cdot \mathbf{R}_{i j}\right)+E_{i j}\left(\mathbf{u}_{i j} \cdot \mathbf{R}_{i j}\right)^{3},
\end{aligned}
$$

where

$$
E_{i j}=-\frac{448 A}{R_{i j}^{18}}+\frac{80 B}{R_{i j}^{12}}
$$

Substituting the expression for $U_{i j}$ from Equation (14) to Equation (11) leads to a representation of the total crystal energy in terms of ascending powers of $\mathbf{u}$ in the form

$$
U=\frac{1}{2} \sum_{i} \sum_{j \neq i} U_{i j}=\frac{1}{2} \sum_{i} \sum_{j \neq i}\left(U_{2, i j}+U_{3, i j}\right)=U_{2}+U_{3},
$$

where $U_{2}=\left(\frac{1}{2} \sum_{i j} U_{2, i j}\right)$ and $U_{3}=\left(\frac{1}{2} \sum_{i j} U_{3, i j}\right)$ contain the quadratic and cubic terms in $u_{i j}$, respectively. For notational simplicity, the self-interaction terms (corresponding to $i=j$ ) in Equation (20) are set to zero, thus allowing the elimination of the extra condition $j \neq i$ henceforth.

The quadratic term $U_{2}$ in (20) may be expressed in Cartesian component form as

$$
\begin{aligned}
U_{2} & =\frac{1}{2} \sum_{i j}\left[C_{i j} u_{i j}^{2}+D_{i j}\left(\mathbf{u}_{i j} \cdot \mathbf{R}_{i j}\right)^{2}\right] \\
& =\frac{1}{2} \sum_{i j} C_{i j}\left[\sum_{\alpha}\left(u_{j}^{\alpha}-u_{i}^{\alpha}\right)^{2}\right]+\frac{1}{2} \sum_{i j} D_{i j}\left[\sum_{\alpha} R_{i j}^{\alpha}\left(u_{j}^{\alpha}-u_{i}^{\alpha}\right)\right]^{2} \\
& =\frac{1}{2} \sum_{i j} C_{i j}\left[\sum_{\alpha}\left(u_{i}^{\alpha}\right)^{2}-2 u_{i}^{\alpha} u_{j}^{\alpha}+\left(u_{j}^{\alpha}\right)^{2}\right]+\frac{1}{2} \sum_{i j} D_{i j}\left[\sum_{\alpha \beta} R_{i j}^{\alpha} R_{i j}^{\beta}\left(u_{i}^{\alpha} u_{i}^{\beta}-2 u_{i}^{\alpha} u_{j}^{\beta}+u_{j}^{\alpha} u_{j}^{\beta}\right)\right] .
\end{aligned}
$$

Since the order of $i$ and $j$ in the sums may be interchanged without altering the values of $C_{i j}$ and $D_{i j}$, it follows that

$$
\sum_{i j, \alpha} C_{i j}\left(u_{j}^{\alpha}\right)^{2}=\sum_{i j, \alpha} C_{j i}\left(u_{i}^{\alpha}\right)^{2}=\sum_{i j, \alpha} C_{i j}\left(u_{i}^{\alpha}\right)^{2}
$$

and

$$
\sum_{i j, \alpha \beta} D_{i j} u_{j}^{\alpha} u_{j}^{\beta}=\sum_{i j, \alpha \beta} D_{j i} u_{i}^{\alpha} u_{i}^{\beta}=\sum_{i j, \alpha \beta} D_{i j} u_{i}^{\alpha} u_{i}^{\beta}
$$


Therefore, it follows from Equation (21) that

$$
U_{2}=\sum_{i j} C_{i j}\left[\sum_{\alpha}\left(u_{i}^{\alpha}\right)^{2}-u_{i}^{\alpha} u_{j}^{\alpha}\right]+\sum_{i j} D_{i j}\left[\sum_{\alpha \beta} R_{i j}^{\alpha} R_{i j}^{\beta}\left(u_{i}^{\alpha} u_{i}^{\beta}-u_{i}^{\alpha} u_{j}^{\beta}\right)\right] .
$$

Comparing the preceding expression to the quadratic term in Equation (2) leads to the representation of the harmonic $\mathrm{FC} \Phi_{i j}^{\alpha \beta}$ for an LJ crystal in the form

$$
\Phi_{i j}^{\alpha \beta}=2\left[\sum_{k}\left(C_{i k} \delta^{\alpha \beta}+D_{i k} R_{i k}^{\alpha} R_{i k}^{\beta}\right) \delta_{i j}-C_{i j} \delta^{\alpha \beta}-D_{i j} R_{i j}^{\alpha} R_{i j}^{\beta}\right],
$$

where $\delta^{\alpha \beta}$ and $\delta_{i j}$ both denote the Kronecker delta symbol. It can be readily concluded that the expression for the harmonic FC in (25) satisfies the Acoustic Sum Rule (ASR), which is a statement of translation-invariance of the potential energy of the crystal [9]. Furthermore, it is also easy to verify that the harmonic FCs automatically satisfy the linearized version of rotational invariance, which is sufficient, insofar as the linear theory of elasticity gives physically reasonable results [10].

A similar procedure may be carried out for the cubic term $U_{3}$ in Equation (20), leading to

$$
\begin{aligned}
U_{3}= & \frac{1}{2} \sum_{i j}\left[D_{i j} u_{i j}^{2}\left(\mathbf{u}_{i j} \cdot \mathbf{R}_{i j}\right)+E_{i j}\left(\mathbf{u}_{i j} \cdot \mathbf{R}_{i j}\right)^{3}\right] \\
= & \frac{1}{2} \sum_{i j} D_{i j}\left[\sum_{\alpha \beta} R_{i j}^{\beta}\left(u_{j}^{\alpha}-u_{i}^{\alpha}\right)^{2}\left(u_{j}^{\beta}-u_{i}^{\beta}\right)\right]+\frac{1}{2} \sum_{i j} E_{i j}\left[\sum_{\alpha} R_{i j}^{\alpha}\left(u_{j}^{\alpha}-u_{i}^{\alpha}\right)\right]^{3} \\
= & \frac{1}{2} \sum_{i j} D_{i j}\left[\sum_{\alpha \beta} R_{i j}^{\beta}\left(\left(u_{i}^{\alpha}\right)^{2}-2 u_{i}^{\alpha} u_{j}^{\alpha}+\left(u_{j}^{\alpha}\right)^{2}\right)\left(u_{j}^{\beta}-u_{i}^{\beta}\right)\right] \\
& +\frac{1}{2} \sum_{i j} E_{i j}\left[\sum_{\alpha \beta \gamma} R_{i j}^{\alpha} R_{i j}^{\beta} R_{i j}^{\gamma}\left(u_{j}^{\alpha}-u_{i}^{\alpha}\right)\left(u_{j}^{\beta}-u_{i}^{\beta}\right)\left(u_{j}^{\gamma}-u_{i}^{\gamma}\right)\right] .
\end{aligned}
$$

Simplifying the preceding expression using the symmetries of $D_{i j}$ and $E_{i j}$ results in

$$
\begin{array}{r}
U_{3}=\sum_{i j} D_{i j}\left[\sum_{\alpha \beta} R_{i j}^{\beta}\left(-\left(u_{i}^{\alpha}\right)^{2} u_{i}^{\beta}+\left(u_{i}^{\alpha}\right)^{2} u_{j}^{\beta}+2 u_{i}^{\alpha} u_{i}^{\beta} u_{j}^{\alpha}\right)\right] \\
+\sum_{i j} E_{i j}\left[\sum_{\alpha \beta \gamma} R_{i j}^{\alpha} R_{i j}^{\beta} R_{i j}^{\gamma}\left(-u_{i}^{\alpha} u_{i}^{\beta} u_{i}^{\gamma}+3 u_{i}^{\alpha} u_{i}^{\beta} u_{j}^{\gamma}\right)\right] .
\end{array}
$$


Comparing (27) to the cubic term in Equation (2) results in an expression for the anharmonic FC $\Psi_{i j k}^{\alpha \beta \gamma}$ for a LJ crystal in the form

$$
\begin{aligned}
\Psi_{i j k}^{\alpha \beta \gamma}=-2[ & \delta_{i j}\left(D_{i k}\left(\delta^{\alpha \beta} R_{i k}^{\gamma}+\delta^{\beta \gamma} R_{i k}^{\alpha}+\delta^{\gamma \alpha} R_{i k}^{\beta}\right)+3 E_{i k} R_{i k}^{\alpha} R_{i k}^{\beta} R_{i k}^{\gamma}\right) \\
+ & \delta_{j k}\left(D_{j i}\left(\delta^{\beta \gamma} R_{j i}^{\alpha}+\delta^{\gamma \alpha} R_{j i}^{\beta}+\delta^{\alpha \beta} R_{j i}^{\gamma}\right)+3 E_{j i} R_{j i}^{\beta} R_{j i}^{\gamma} R_{j i}^{\alpha}\right) \\
& \left.+\delta_{k i}\left(D_{k j}\left(\delta^{\gamma \alpha} R_{k j}^{\beta}+\delta^{\alpha \beta} R_{k j}^{\gamma}+\delta^{\beta \gamma} R_{k j}^{\alpha}\right)+3 E_{k j} R_{k j}^{\gamma} R_{k j}^{\alpha} R_{k j}^{\beta}\right)\right]
\end{aligned}
$$

It may be again verified that this expression for the cubic FC satisfies the ASR as generalized to higher-order FCs [3].

From Equations (25) and (28), it can be seen that the FCs between an atom and its neighbors depend on the components of the relative equilibrium positions. To compute the FCs for a solid LJ crystal, a supercell needs to be considered with all atoms at their equilibrium positions so that the FCs between the central atom and all other atoms in the supercell are determined according to Equations (25) and (28). This supercell should be large enough to take into account all the significant interatomic interactions between any two atoms of the LJ crystal. This condition is satisfied if the length of the supercell along each Cartesian direction is at least twice the cutoff distance for interatomic interactions. For LJ solids, it is sufficient to take the cutoff distance to be greater than $2.5\left(\frac{A}{B}\right)^{1 / 6}[2]$.

\section{Simulation procedure}

The proposed technique is implemented for a crystal lattice in LAMMPS [11], a widely used general-purpose classical molecular dynamics code which runs on a multitude of serial and parallel computer architectures.

\subsection{Implementation as an interatomic potential}

The primary utility of any interatomic potential is in the computation of the total potential energy of the system and the total force on every atom at each time-step. For the truncated Taylor series potential, these quantities are computed according to Equations (2) and (3), respectively. The FCs appearing in these equations are obtained simply by accessing a lookup table of FCs, which may be generated analytically, according to the formulae in Section 3, or by ab initio methods (e.g., based on DFT simulations). This lookup table must provide the values of the FCs between an atom and each of its neighbors. 
Unlike a conventional LJ potential, which depends exclusively on the current distance between atoms, the truncated Taylor series potential of Equation (2) depends on the displacement of atoms from their equilibrium lattice positions. Since most MD codes do not store the equilibrium positions, these need to be extracted from information contained in the current atomic positions. This is possible as long as assumption (4) holds true, since, in that case, the equilibrium position of an atom is the nearest lattice site. The resulting map of atoms to lattice sites is represented by a table, termed here "TableID".

This approach is analogous to the total Lagrangian finite element formulation in continuum mechanics [12]. The algorithm implemented in the interatomic potential consists of the following key steps:

1. At each time-step, the equilibrium lattice position of every atom, which is the nearest lattice site to its current position, is identified.

2. For each atom, a list of all of its neighbors within a given cutoff distance is compiled by a low-cost binary search, such as binning. This is a standard feature available in all MD codes.

3. For each neighbor of a given atom, its TableID is determined with respect to the given atom using the lattice equilibrium positions of the two atoms.

4. TableID is used to identify the appropriate FCs from the lookup table for computing the force on a given atom due to each of its neighbors.

5. The resultant force on each atom and the total energy of the crystal lattice are computed according to Equations (2) and (3), in which the FCs for the case of the LJ solid are computed using equations (25) and (28).

The algorithm to determine the equilibrium lattice position of every atom is detailed in the Appendix.

\subsection{Computation of specific heat capacity for solid Ar}

To assess the numerical accuracy of the proposed technique, solid Ar is chosen as a test material. Ar (atomic mass $=39.948 \mathrm{gm} / \mathrm{mol}$ ) is well-described with the LJ potential with the LJ parameters $\epsilon=0.0104 \mathrm{eV}$ and $\sigma=3.40 \AA[5]$.

As argued in Section 2.2, the temperature range within which condition (4) remains valid depends on the width of the potential well at equilibrium. For solid Ar modeled 
using LJ with the potential function truncated at third order it was observed that the MD simulations become unstable at temperatures above $10 \mathrm{~K}$. The instability is due to the thermal motion of atoms past a critical distance from their equilibrium positions at some point of time during the simulation.

The crystal structure of solid Ar is a face-centered cubic (FCC) lattice with one basis atom per lattice site. The lattice constant for FCC Ar is determined by performing NPT simulations at a constant pressure of 1 bar and at temperatures ranging from $2 \mathrm{~K}$ to $10 \mathrm{~K}$, as discussed in [13]. All of the MD simulations were performed with a time-step of $4 \mathrm{fs}$, which is found to be sufficient to resolve all the lattice vibrations, and cutoff of $3.1 \sigma$ [13].

The specific heat of solid Ar may be estimated from MD simulations using a formula which relates it to the fluctuations of the total energy of the system under thermodynamic equilibrium in a canonical (i.e., NVT) ensemble according to

$$
C_{v}=\frac{\left\langle\left(\delta E^{2}\right)\right\rangle}{k_{B} T^{2}}
$$

see, e.g., [14]. Here, $\left\langle\left(\delta E^{2}\right)\right\rangle$ denotes the ensemble average of the mean square deviation of the total energy of the system in the NVT ensemble, $k_{B}$ the Boltzmann constant, and $T$ the temperature of the simulation. The FCs used in the computation of energy are taken from a lookup table based on Equations (25) and (28). This table is constructed outside the MD code and contains all harmonic and anharmonic FCs generated between the central atom and all other atoms in a supercell whose size is at least twice the typical LJ cutoff distance.

A Nosé-Hoover thermostat $[15,16]$ is employed in the NVT system to ensure that when the temperature of the system reaches beyond a certain window around the target temperature it is restored back to the target temperature by the application of appropriate fictitious forces. One of the critical thermostat parameters in an NVT simulation is the rate $\zeta$ at which the system is restored to the target temperature, as this determines the magnitude of the fluctuations in the total energy of the system at equilibrium. Since the computed value of the heat capacity and other thermophysical properties determined from equilibrium fluctuations (e.g., thermal conductivity using the Green-Kubo formula [17]) depend on the size of these fluctuations, the thermostat parameter $\zeta$ should be set to an appropriate value while performing MD simulations in an NVT ensemble. To determine the appropriate value of $\zeta$ for the NVT ensemble of LJ Ar, a pure harmonic crystal of solid $\mathrm{Ar}$ is considered, for which the heat capacity $C_{v}$ is derived by the Dulong-Petit law as

$$
C_{v}^{h}=3 N k_{B},
$$


where $N$ denotes the total number of atoms in the system [18]. The pure harmonic crystal is simply realized by using the truncated Taylor series interatomic potential, where the cubic FCs are set to zero. MD simulations are then performed in the NVT ensemble for different values of $\zeta$ and different temperatures and the resulting values of $C_{v}$ are compared to the Dulong-Petit value. The value of $\zeta$ that yields the closest match to the theoretical estimate is selected for all subsequent MD simulations in NVT ensembles (see also Section $5)$.

\subsection{Computation of thermal conductivity for solid Ar}

To test the efficiency of this method in capturing anharmonicity, a transport property such as the bulk thermal conductivity $\kappa$ may be employed, as its value depends strongly on the anharmonic terms in the potential energy. The thermal conductivity of a material is computed from an NVT ensemble using the Green-Kubo formula [19]

$$
\kappa=\frac{1}{3 k_{B} V T^{2}} \int_{0}^{\infty}\left\langle\tilde{\mathbf{J}}_{Q}(0) \cdot \tilde{\mathbf{J}}_{Q}(t)\right\rangle d t
$$

where $\tilde{\mathbf{J}}_{Q}$ is the heat flux in the system under thermodynamic equilibrium in a canonical ensemble and $\langle\cdot\rangle$ denotes ensemble average. In practice, the upper limit of the integral in Equation (31) is replaced by a finite time $\left(\tau_{C}\right)$, which must be taken to be long enough for the correlations between the heat flux to decay to nearly zero in that time interval [20].

The relative error in the computed value of a transport property computed by the Green-Kubo method is given by

$$
\frac{\Delta \kappa}{\kappa} \leq 2 \sqrt{\frac{\tau_{C}}{\tau}}
$$

where $\tau$ denotes the total number of time-steps during the simulation [21]. Hence, having chosen a suitable correlation time $\tau_{C}$, the total simulation time for a desired accuracy $a=\frac{\Delta \kappa}{\kappa}$ is given by

$$
\tau \geq \frac{4 \tau_{C}}{a^{2}} .
$$

Using this result, it is possible to estimate the minimum simulation time required to obtain the thermal conductivity to any desired accuracy.

\section{Results}

Each MD simulation was run for 7,000,000 time steps and each of the results obtained are averaged over an ensemble of 10 independent runs, while the errors reported are taken 
to be the standard deviation in the result over the 10 runs. Figure 3 shows a plot of the specific heat capacity obtained from MD simulations on pure harmonic Ar in an NVT ensemble. The specific heat capacity (including error bars indicating its standard deviation) is computed using Equation (29) for temperatures ranging from 2 to $10 \mathrm{~K}$ and for different values of $\zeta$. A comparison of the computed values of $C_{v}$ to the one obtained from the Dulong-Petit law shows that beyond a certain value of $\zeta, C_{v}$ is fairly close to the expected value. Of the three values of $\zeta$ that yield the smallest deviation of the average value of $C_{v}$ from (30), the optimal ( $\zeta=8 \mathrm{ps}$ ) is selected to be the one that minimizes the standard deviation.

Using the optimal value of $\zeta$, MD simulations are performed for a solid crystal of Ar using the standard LJ potential and the one obtained from the Taylor series based potential truncated at third order for different unit cell sizes of 4,5 and 6 unit cells in each direction to confirm size convergence. It was found that by the difference between the values of $C_{v}$ computed for these different unit cell sizes converged to within $10 \%$ by 6 unit cells. This showed that size convergence had been attained by the largest domain size. Figure 4 shows the values of the $C_{v}$ for 6 unit cells. The figure demonstrates that the maximum deviation between the computed values of $C_{v}$ for the two potentials across all temperatures from 2 to $10 \mathrm{~K}$ is less than $10 \%$.

The difference in the specific heat capacities of the pure harmonic crystal and the LJ crystal is due to the anharmonicity in the latter [5, Chapter 22]. Thus, the excellent agreement between the values of $C_{v}$ for the standard LJ potential and its truncated counterpart shows that, within the given temperature range, the cubic terms are sufficient to capture the anharmonicity of the LJ Ar crystal.

To confirm the effectiveness of this method in capturing the anharmonicity of LJ Ar, the thermal conductivity for solid Ar obtained for the truncated potential is compared to that obtained for the standard LJ potential. To this end, first the correlation time to be used as the upper limit of the integral in Equation (31) is determined by noting the value at which the heat flux autocorrelation function decays to zero. The correlation time is not a constant but depends on the temperature of the simulation and must be determined by trial and error. For instance, it was found that at $2 \mathrm{~K}, \tau_{C}$ must be taken to be $1024 \mathrm{ps}$, whereas at $6 \mathrm{~K}$, it is sufficient to take $\tau_{C}$ to be $256 \mathrm{ps}$. Then, the total number of time-steps required to be performed for the relative error in the computed value of thermal conductivity to be about $10 \%$ is determined according to (33). This procedure is repeated for the same unit cell sizes in order to ensure convergence with respect to domain size to within $10 \%$. 
Figure 5 shows the decay of the heat flux autocorrelation function $\left\langle\tilde{\mathbf{J}}_{Q}(0) \cdot \tilde{\mathbf{J}}_{Q}(t)\right\rangle$ with correlation time for the truncated Taylor series-based potential at $6 \mathrm{~K}$ for 6 unit cells, while Figure 6 shows the integral of the same autocorrelation function with respect to the correlation time. The difference between the two conductivity estimates in 6 is due to the Taylor series truncation relative to the standard LJ potential. Figure 7 demonstrates that the maximum deviation between the computed values of $\kappa$ for the two potentials across all temperatures from 2 to $10 \mathrm{~K}$ is less than $20 \%$.

\section{Conclusion}

A new method for determining and implementing a truncated interatomic potential using a Taylor series expansion of any general potential has been detailed. The coefficients in this expansion may be derived analytically, as illustrated in this paper for the case of LJ Ar or, in the case of real materials where the interatomic potential is not known, through $a b$ initio methods such as DFT simulations. Restrictions in the use of the truncated potential (evidenced by limits on the temperature range) are shown to arise from the existence of critical atomic displacements beyond which the resulting material model becomes unstable, as illustrated in Figure 1. Thus, there exists a range of temperatures for which the proposed method is readily applicable. The practical limitation in determining higher-order FCs in the Taylor series expansion of the crystal potential energy necessitates the investigation of how many higher-order terms are required to accurately estimate thermophysical properties such as heat capacity and thermal conductivity that depend on the higher-order terms of the crystal potential energy. Figure 4 shows that for LJ Ar, the method of truncated Taylor series potential produces a reasonably accurate estimate of the heat capacity, which shows its effectiveness in modeling a bulk equilibrium property. To further test the utility of this method, it is also shown for LJ Ar that the effect of anharmonicity in thermal conductivity may be well captured by merely truncating the Taylor series at third order, as can be seen from Figures 6 and 7. The extension of this method to the estimation of thermophysical properties of other materials that require ab initio methods such as DFT to compute the FCs will be treated in a subsequent article. In this case, the determination of the error incurred in the estimation of thermophysical properties using the truncated Taylor series method is not computationally possible, and so the comparison must be done with experimental results. In cases where no experimental data may be available, the technique of the truncated Taylor series potential provides a valuable computational tool 
to predict the properties of thermophysical phenomena.

\section{Acknowledgements}

The authors would like to thank Professor Christos Papadimitrou of the Department of Electrical Engineering and Computer Sciences at the University of California, Berkeley for his help with the algorithm for computing equilibrium positions in a crystal lattice. This work used the Extreme Science and Engineering Discovery Environment (XSEDE), which is supported by National Science Foundation grant number ACI-1053575 [22].

\section{References}

[1] A.J.H. McGaughey and J.M. Larkin. Predicting phonon properties from equilibrium molecular dynamics simulations. Ann. Rev. Heat Transfer, 17:49-87, 2014.

[2] A.J.H. McGaughey and M. Kaviany. Phonon transport in molecular dynamics simulations: Formulation and thermal conductivity prediction. Adv. Heat Transfer, 39:169$255,2006$.

[3] K. Esfarjani and H. Stokes. Method to extract anharmonic force constants from first principles. Phys. Rev. B, 77(14):1441121-7, 2008.

[4] K. Esfarjani, G. Chen, and H. Stokes. Heat transport in silicon from first-principles calculations. Phys. Rev. B, 84:0852041-11, 2011.

[5] N.D. Ashcroft and N.W. Mermin. Solid State Phys. Thomson Learning, New York, 1976.

[6] J.H. Irving and G. Kirkwood. The statistical mechanics theory of transport processes. iv the equations of hydrodynamics. J. Chem. Phys., 18(06):817-829, 1950.

[7] K. K. Mandadapu. Homogeneous Non-Equilibrium Molecular Dynamics Methods for Calculating the Heat Transport Coefficient of Solids and Mixtures. PhD thesis, University of California, Berkeley, 2011.

[8] K.K. Mandadapu, R.E. Jones, and P. Papadopoulos. Generalization of the homogeneous nonequilibrium molecular dynamics method for calculating thermal conductivity to multibody potentials. Phys. Rev. E, 80(4):047702, 2009.

[9] R.M. Pick, M.H. Cohen, and R.M. Martin. Microscopic theory of force constants in the adiabatic approximation. Phys. Rev. B, 1:910-920, 1970.

[10] G. Leibfried and W. Ludwig. Gleichgewichtsbedingungen in der Gittertheorie. Zeitschrift für Physik, 160(1):80-92, 1961. 
[11] S. Plimpton. Fast parallel algorithms for short-range molecular dynamics. J. Comp. Phys., 117(1):1-19, 1995.

[12] K.-J. Bathe. Finite Element Procedures. Prentice-Hall, Englewood-Cliffs, 1996.

[13] A.J.H. McGaughey and M. Kaviany. Thermal conductivity decomposition and analysis using molecular dynamics simulations. Part I. Lennard-Jones argon. Int. J. Heat and Mass Transfer, 47:1783-1798, 2004.

[14] D. Chandler. Introduction to Modern Statistical Mechanics. Oxford University Press, New York, USA, 1987.

[15] S. Nosé. A molecular dynamics method for simulations in the canonical ensemble. Mol. Phys., 52(2):255-268, 1984.

[16] W. G. Hoover. Canonical dynamics: Equilibrium phase-space distributions. Phys. Rev. A, 31:1695-1697, Mar 1985.

[17] D. J. Evans and G. Morriss. Statistical Mechanics of Nonequilibrium Liquids. Cambridge University Press, second edition, 2008. Cambridge Books Online.

[18] A.-T. Petit and P.-L. Dulong. Recherches sur quelques points importants de la théorie de la chaleur. Ann. Chm. Phys., 10:395-413, 1819.

[19] R. Kubo, M. Yokota, and S. Nakajima. Statistical-mechanical theory of irreversible processes - II: Response to thermal disturbance. J. Phys. Soc. Jpn., 12:1203-1211, 1957.

[20] M.P. Allen and D.J. Tildesley. Computer Simulation of Liquids. Oxford University Press, New York, USA, 1989.

[21] R.E. Jones and K.K. Mandadapu. Adaptive Green-Kubo estimates of transport coefficients from molecular dynamics based on robust error analysis. J. Chem. Phys., 136(15), 2012.

[22] J. Towns, T. Cockerill, M. Dahan, I. Foster, K. Gaither, A. Grimshaw, V. Hazlewood, S. Lathrop, D. Lifka, G. D Peterson, R. Roskies, J.R. Scott, and N. WilkinsDiehr. XSEDE: Accelerating scientific discovery. Computing in Science $\mathscr{E}$ Engineering, 16(5):62-74, 2014.

[23] D. Micciancio and S. Goldwasser. Complexity of Lattice Problems: A Cryptographic Perspective, chapter Closest Vector Problem, pages 45-68. Springer, Boston, 2002.

\section{Appendix A: An efficient algorithm for computing equilib- rium positions in a crystal lattice}

The problem of computing the nearest equilibrium lattice position of an atom is a special case of the Closest Vector Problem (CVP) for three dimensions [23]. 
Any crystal lattice in three dimensions may be completely specified by listing its basis vectors $\mathbf{a}_{1}, \mathbf{a}_{2}$ and $\mathbf{a}_{3}$ and the positions $\mathbf{b}_{i}(i=1, \ldots, N)$ of the basis atoms within a unit cell. Then, the position of any lattice site is given by

$$
\mathbf{R}=m_{1} \mathbf{a}_{1}+m_{2} \mathbf{a}_{2}+m_{3} \mathbf{a}_{3}+\mathbf{b}_{i}
$$

where $m_{1}, m_{2}$ and $m_{3}$ denote some integer values.

To describe the algorithm succinctly, let $\mathbf{A}$ denote the matrix whose columns are the components of the lattice basis vectors $\mathbf{a}_{1}, \mathbf{a}_{2}$ and $\mathbf{a}_{3}$. The problem is to find the nearest lattice site to a given arbitrary position vector $\mathbf{r}$. Let $m_{1}, m_{2}$ and $m_{3}$ denote integers representing the unit cell of the lattice site $\mathbf{R}$ nearest to the current position $\mathbf{r}$. The algorithm consists of the following steps:

1. For each basis atom $i$, solve the linear system of equations given by

$$
\mathbf{r}=\mathbf{A n}+\mathbf{b}_{i}
$$

where $\mathbf{n}=\left[n_{1}, n_{2}, n_{3}\right]^{T}$ represents the solution to the aforementioned linear system. Note that $\mathbf{n}$ will have non-integral components in general.

2. Set $m_{i}$ to the nearest integer to the corresponding $n_{i}$ by rounding for $i=1,2,3$ and set $\mathbf{R}=\sum_{i} \mathbf{A} \mathbf{m}+\mathbf{b}_{i}$, where $\mathbf{m}=\left[m_{1}, m_{2}, m_{3}\right]^{T}$ is the vector containing the integer values $m_{1}, m_{2}, m_{3}$, which represent the tentative unit cell of the lattice site $\mathbf{R}$ nearest to the current position $\mathbf{r}$.

3. If the distance between $\mathbf{r}$ and $\mathbf{R}$ is less than half the nearest-neighbor distance in the crystal lattice, then $\mathbf{R}$ is the desired nearest equilibrium lattice position to the current position.

4. If the aforementioned condition is not satisfied, then a series of parallelopipeds of increasing sizes centered at $\mathbf{R}$ are constructed. For each parallelopiped, the distance between each lattice site on the surface of the parallelopiped and $\mathbf{r}$ is computed and the smallest distance for that parallelopiped is noted. If this smallest distance is larger than the smallest distance between $\mathbf{r}$ and a lattice site found so far, this means that the parallelopiped completely envelopes the sphere with center at $\mathbf{r}$ and radius equal to this shortest distance. This implies that no parallelopiped of larger size can contain the nearest lattice site on its surface and so the search is terminated with the lattice site $\mathbf{R}$ giving the shortest distance so far. 
This algorithm is found to be very efficient in practice as it always terminates within two to three steps. 


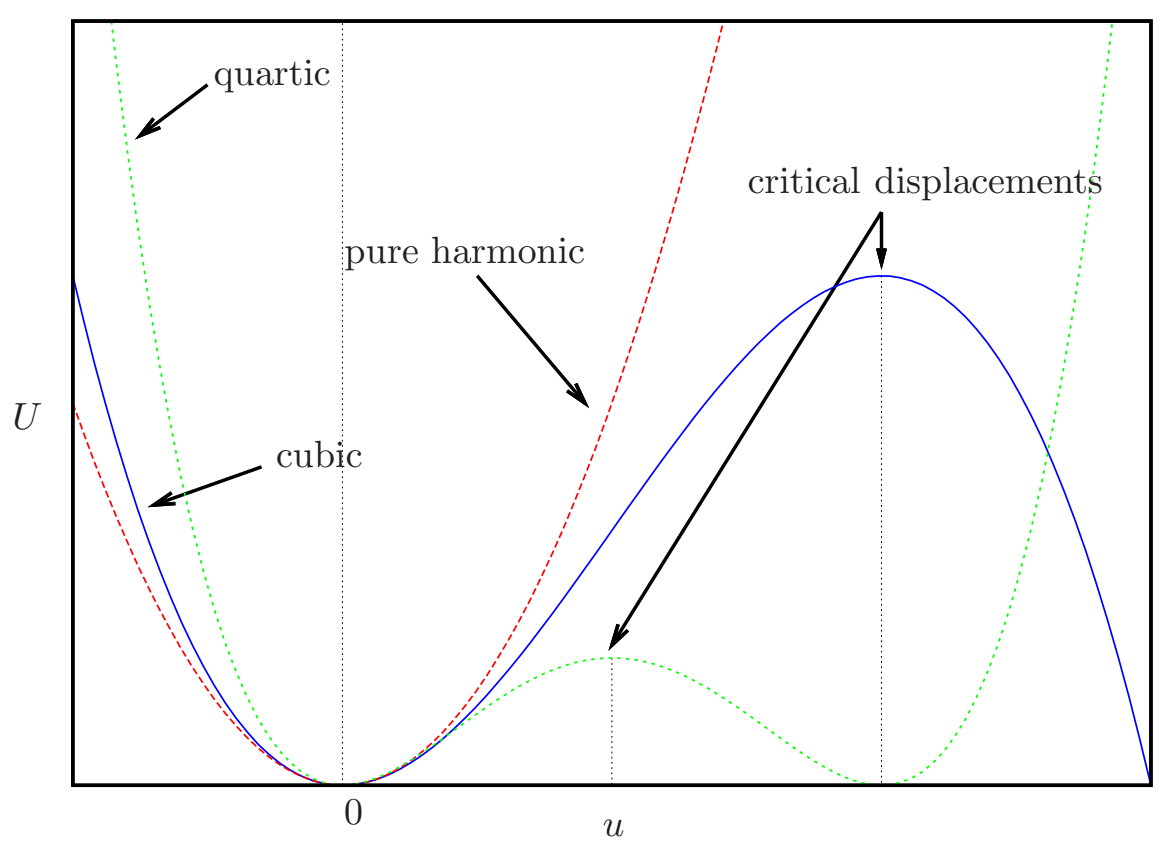

Figure 1: Schematic depiction of a pure harmonic and anharmonic potentials derived from Taylor series expansion of a general crystal potential energy 


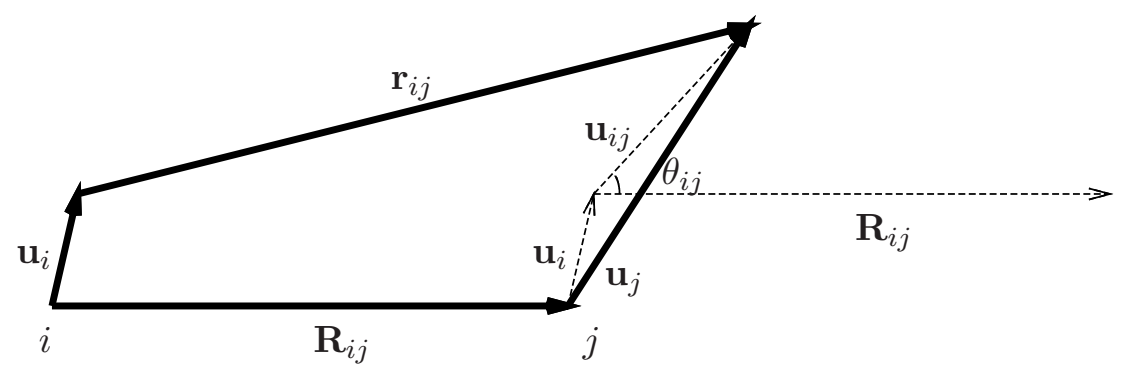

Figure 2: Atomic displacements from equilibrium position 


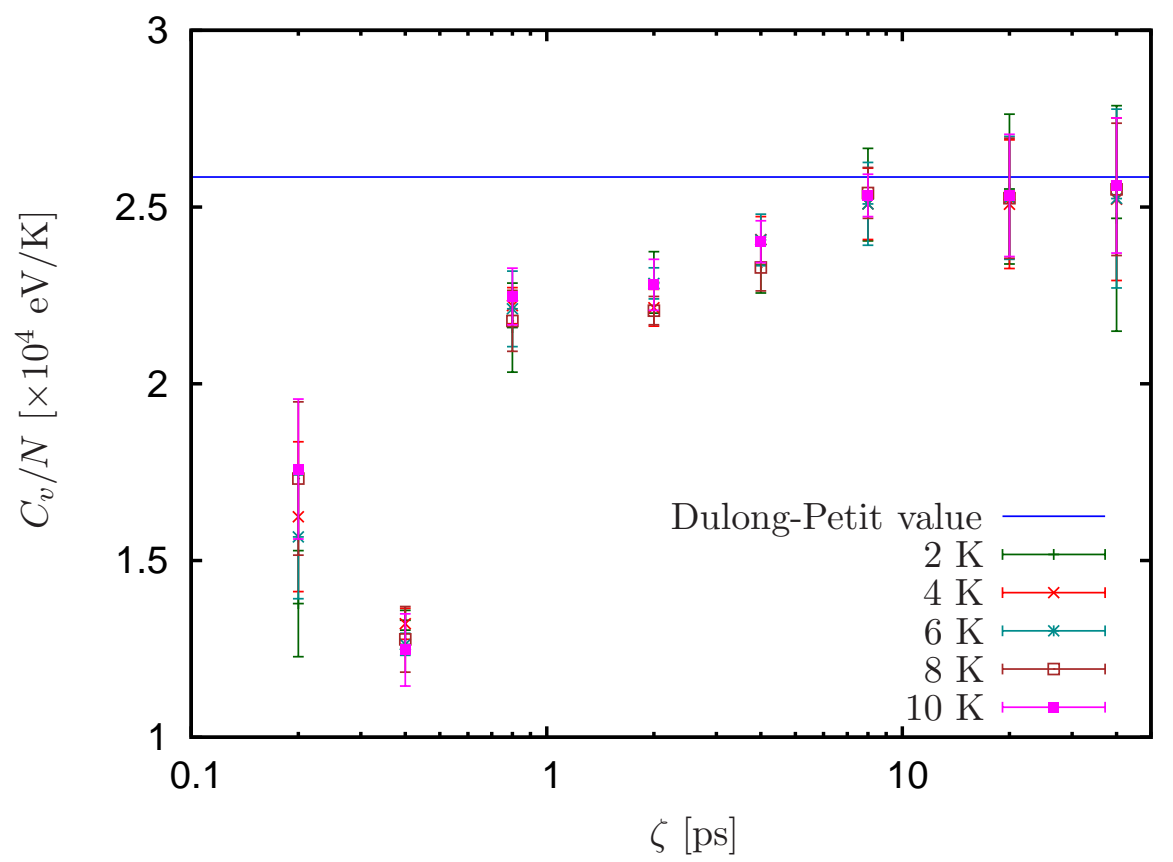

Figure 3: Variation of specific heat capacity with thermostat parameter $\zeta$ in NVT ensemble 


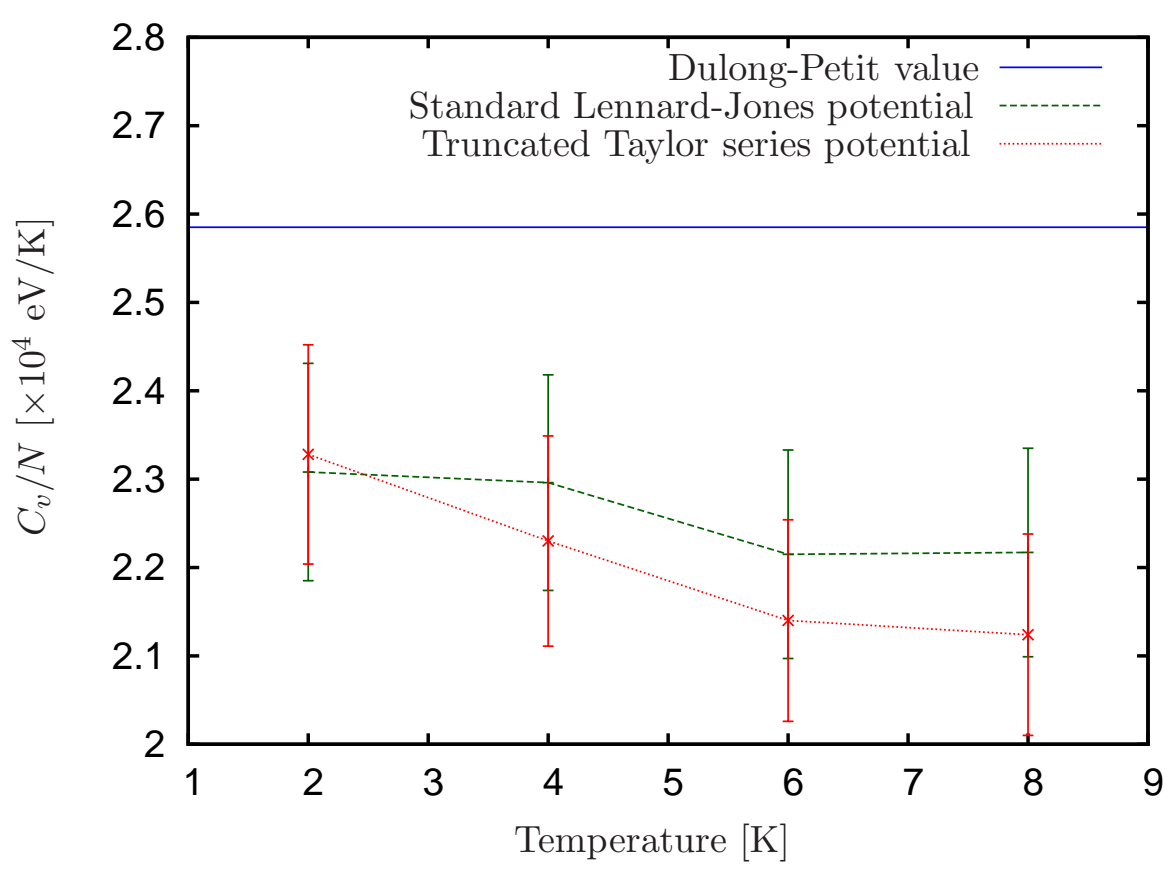

Figure 4: Comparison of specific heat capacity for standard LJ and truncated Taylor series based potentials for solid Ar at different temperatures 


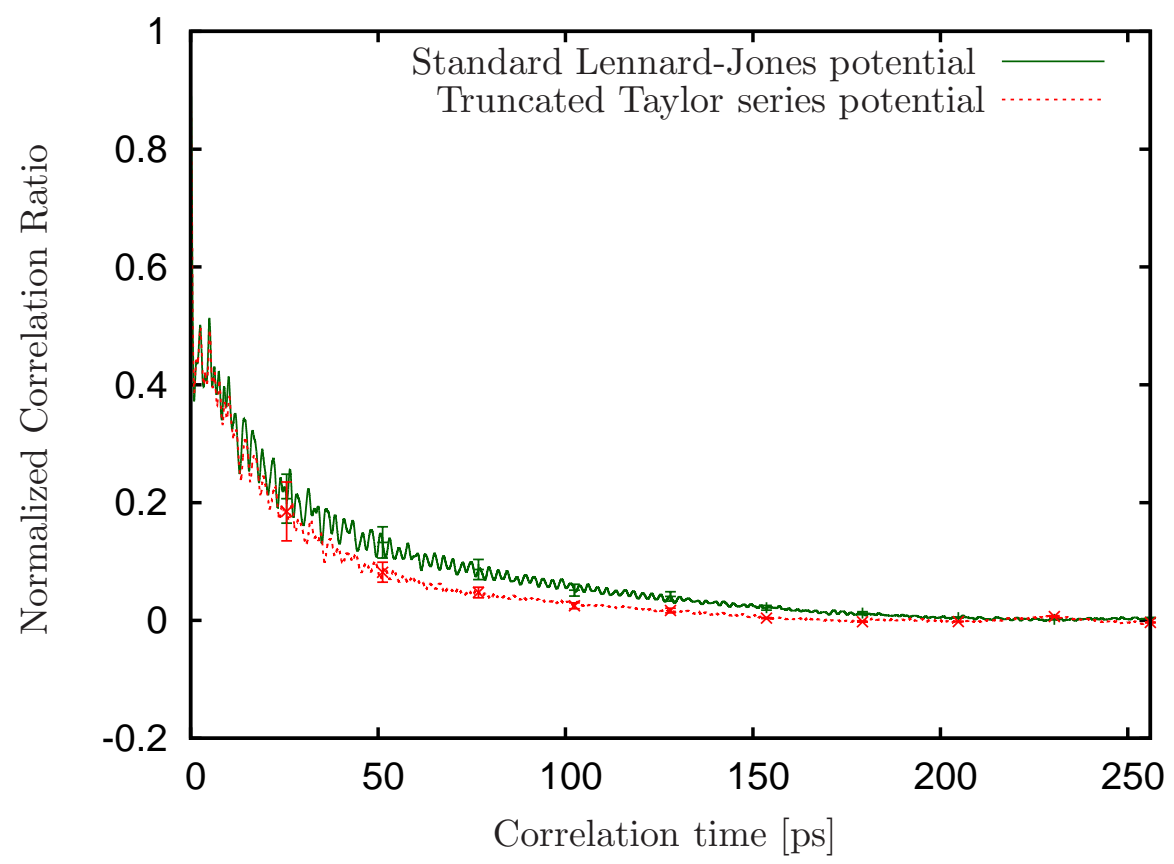

Figure 5: Decay of the heat flux autocorrelation function for the standard LJ potential and the truncated Taylor series-based potential with correlation time at $6 \mathrm{~K}$ for a $6 \times 6 \times 6$ unit cells 


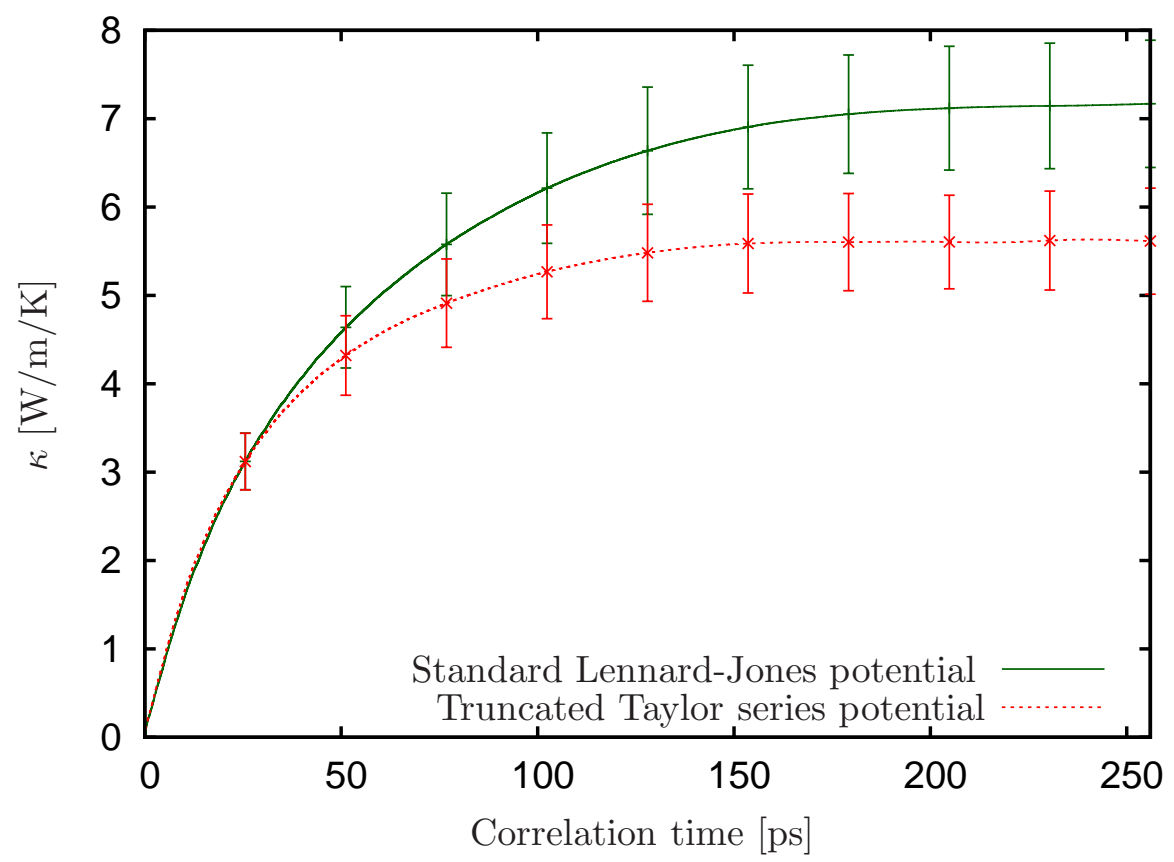

Figure 6: Integral of the heat flux autocorrelation function for the standard LJ potential and the truncated Taylor series-based potential with respect to correlation time at $6 \mathrm{~K}$ for a $6 \times 6 \times 6$ unit cells 


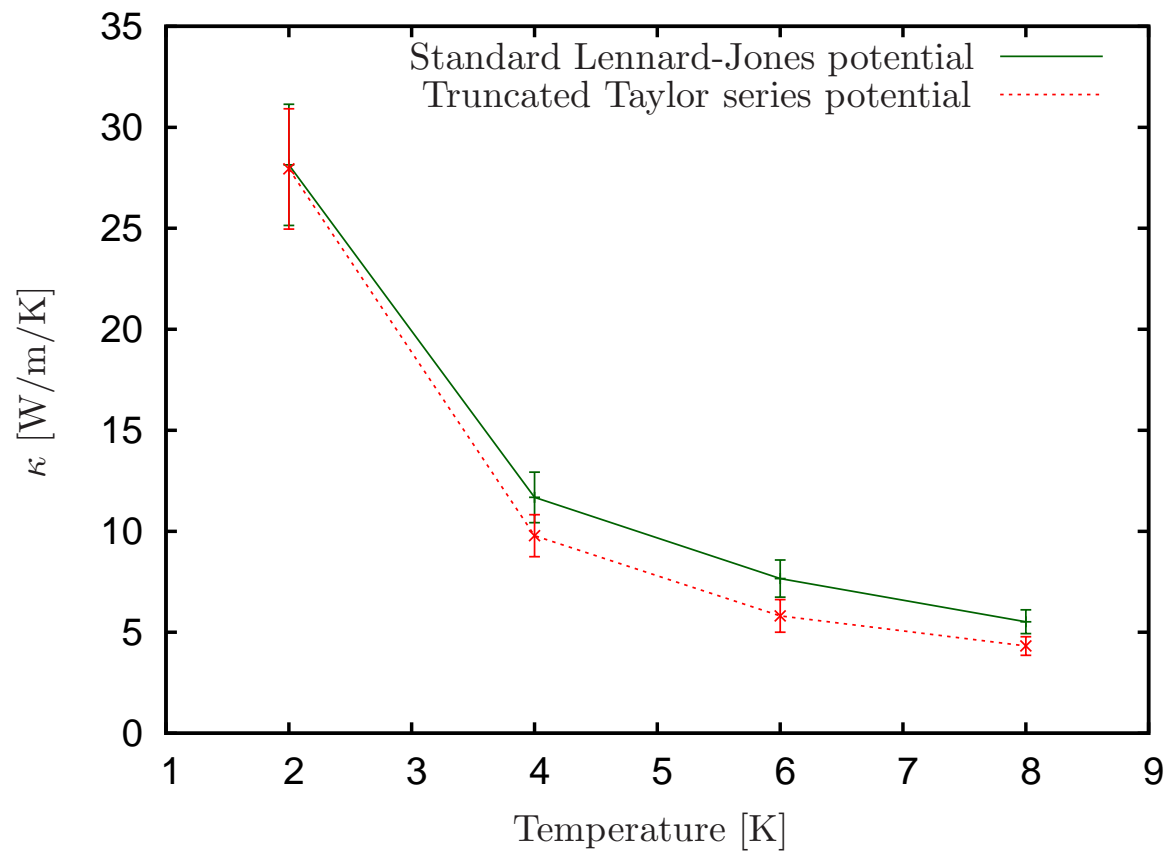

Figure 7: Comparison of thermal conductivity for standard LJ and the truncated Taylor series based potentials for solid Ar at different temperatures for $6 \times 6 \times 6$ unit cells 